\title{
Towards a better understanding of the many facets of attention-deficit/hyperactivity disorder
}

\author{
Pieter J. Hoekstra ${ }^{1}$ \\ Published online: 7 March 2018 \\ c) Springer-Verlag GmbH Germany, part of Springer Nature 2018
}

Attention-deficit/hyperactivity disorder (ADHD) is the most frequently diagnosed mental disorder in children and adolescents and topic of a wide range of scientific publications. Heterogeneity is a key characteristic of ADHD [1]. This is true for many aspects of the disorder. ADHD is highly variable with regard to degree of impairment, associated comorbidities, symptom presentation, response to treatment, and etiology. This month's issue contains no less than six ADHD-related articles, which together deal with a variety of important aspects of ADHD.

Currently, stimulant medication and parent management training represent the mainstream of ADHD treatments, but its long-term effectiveness leaves considerable room for improvement. That is why studies evaluating the effectiveness of alternative treatments are particularly welcome. Two of this month's articles concern such randomized controlled treatment trials. The study by Wennberg and colleagues investigated the effects of training in time-processing ability which included the use of time-assistive devices in an attempt to address the clinically well-known problems with time and timing that may impair daily routines, academic functioning, and social relations [2]. Training lasted about 12 weeks, was tailored to the individual needs of the child, and was oriented at skills readily relevant for daily functioning. Examples include structuring the physical environment to enable organizing clothes and sportswear so that they become easy to find, make sure there are clocks visible in all rooms at home, or let the child compare the amount of time needed to perform the same activity in two different ways, e.g., to walk to school or to bike to school. Time-processing ability was significantly improved in the children who had received the training as compared to the control treatment.

Pieter J. Hoekstra

p.hoekstra@accare.nl

1 Department of Child and Adolescent Psychiatry, University Medical Center Groningen, University of Groningen, Groningen, The Netherlands
Although effect sizes were relatively small, the results highlight the potential of time-skill training to improve daily life functioning in school-age children with ADHD.

The other randomized controlled trial, into the effects of omega-3 supplementation as a treatment for children with ADHD, is a rare example of the placebo condition doing significantly better than the active treatment, even with a clinically relevant effect size [3]. Unfortunately, studies reporting negative results are much less likely to make it into a journal and the fact that data collection for the study had been completed already 7 years ago probably indicates difficulties of getting the paper published. Still, the study is very worthwhile as it has one of the largest samples sizes in this area and was conducted in a double-blinded fashion. The results make one wonder, as the authors appropriately conclude, whether it is worth continuing to perform clinical trials in this field. It certainly suggests that there is no evidence base to apply omega-3 supplementation as a treatment for ADHD in clinical practice.

There is some debate about the precise benefits of methylphenidate treatment for children with ADHD [4] and to what extent prescription of methylphenidate in clinical practice is in line with clinical recommendations. Two of this issue's articles shed light on these important topics around methylphenidate treatment. A study based on Taiwan's large National Health Insurance Research Database clearly indicates protective effects of methylphenidate in reducing traumatic brain injuries, with larger protective effects in relation to increased dosages [5]. It adds to studies pointing to beneficial effects of methylphenidate, e.g., in reducing the risk of substance abuse [6]. The study of Courtabessis et al. [7] in this issue is reassuring in that it indicates that clinicians' treatment decision-making with regard to initiating methylphenidate treatment in a clinic in Montpellier, France, complies well with European clinical guidelines.

Finally, two articles highlight the importance of paying attention to co-occurring problems in ADHD that go beyond well-known associated comorbidities such as 
oppositional-defiant and conduct disorder. A systematic review and meta-analysis in this issue demonstrates that there is a positive association between ADHD and migraine, but not with tension-type headache [8]. This warrants further studies into possibly shared etiological mechanisms between migraine and ADHD. The last ADHD-related article [9] that I would like to highlight reports surprisingly high rates of significant sleep problems in children with ADHD, i.e., in $75 \%$ of girls and over half of boys. Interestingly, specific types of sleep problems appeared to be uniquely related to comorbid conditions such as anxiety symptoms, depression, and oppositional-defiant disorder. As impaired sleep clearly affects day time functioning the article has great relevance for clinical practice. It underlines the importance of systematically assessing each child with ADHD on a broad range of possible co-occurring problems and to carefully plan individualized multimodal treatment.

\section{References}

1. Segenreich D, Paez MS, Regalla MA, Fortes D, Faraone SV, Sergeant J, Mattos P (2015) Multilevel analysis of ADHD, anxiety and depression symptoms aggregation in families. Eur Child Adolesc Psychiatry 24(5):525-536. https://doi.org/10.1007/s0078 7-014-0604-1

2. Wennberg B et al (2017) Effectiveness of time-related interventions in children with ADHD aged 9-15 years: a randomized controlled study. Eur Child Adolesc Psychiatry. https://doi. org/10.1007/s00787-017-1052-5

3. Cornu C et al (2017) A double-blind placebo-controlled randomised trial of omega-3 supplementation in children with moderate ADHD symptoms. Eur Child Adolesc Psychiatry. https://doi. org/10.1007/s00787-017-1058-Z

4. Hoekstra PJ, Buitelaar JK (2016) Is the evidence base of methylphenidate for children and adolescents with attention-deficit/ hyperactivity disorder flawed? Eur Child Adolesc Psychiatry 25(4):339-340. https://doi.org/10.1007/s00787-016-0845-2

5. Liao YT et al (2017) Dosage of methylphenidate and traumatic brain injury in ADHD: a population-based study in Taiwan. Eur Child Adolesc Psychiatry. https://doi.org/10.1007/s0078 7-017-1042-7

6. Groenman AP, Oosterlaan J, Rommelse NN, Franke B, Greven CU, Hoekstra PJ, Hartman CA, Luman M, Roeyers H, Oades RD, Sergeant JA, Buitelaar JK, Faraone SV (2013) Stimulant treatment for attention-deficit hyperactivity disorder and risk of developing substance use disorder. Br J Psychiatry 203(2):112-119

7. Courtabessis E et al (2017) Clinical factors associated with decision to recommend methylphenidate treatment for children with ADHD in France. Eur Child Adolesc Psychiatry. https://doi. org/10.1007/s00787-017-1061-4

8. Salem H et al (2017) ADHD is associated with migraine: a systematic review and meta-analysis. Eur Child Adolesc Psychiatry. https://doi.org/10.1007/s00787-017-1045-4

9. Becker SP et al (2017) The impact of comorbid mental health symptoms and sex on sleep functioning in children with ADHD. Eur Child Adolesc Psychiatry. https://doi.org/10.1007/s0078 7-017-1055-2 